\title{
A bacterial riboswitch class senses xanthine and uric acid to regulate genes associated with purine oxidation
}

\author{
DIANE YU1 and RONALD R. BREAKER ${ }^{1,2,3}$ \\ ${ }^{1}$ Department of Molecular, Cellular and Developmental Biology, Yale University, New Haven, Connecticut 06520-8103, USA \\ ${ }^{2}$ Department of Molecular Biophysics and Biochemistry, Yale University, New Haven, Connecticut 06520-8103, USA \\ ${ }^{3}$ Howard Hughes Medical Institute, Yale University, New Haven, Connecticut 06520-8103, USA
}

\begin{abstract}
Dozens of candidate orphan riboswitch classes have been discovered previously by using comparative sequence analysis algorithms to search bacterial genomic sequence databases. Each orphan is classified by the presence of distinct conserved nucleotide sequences and secondary structure features, and by its association with particular types of genes. One previously reported orphan riboswitch candidate is the "NMT1 motif," which forms a hairpin structure with an internal bulge that includes numerous highly conserved nucleotides. This motif associates with genes annotated to encode various dioxygenase enzymes, transporters, or proteins that have roles associated with thiamin or histidine metabolism. Biochemical evaluation of numerous ligand candidates revealed that NMT1 motif RNA constructs most tightly bind 8-azaxanthine, xanthine, and uric acid, whereas most other closely related compounds are strongly rejected. Genetic assays revealed that NMT1 motif RNAs function to turn off gene expression upon ligand binding, likely by regulating translation initiation. These results suggest that NMT1 motif RNAs function as aptamer domains for a riboswitch class that specifically responds to high concentrations of oxidized purines. Members of this "xanthine riboswitch" class appear to regulate genes predominantly related to purine transport and oxidation, thus avoiding the effects of overproduction of these common purine derivatives.
\end{abstract}

Keywords: aptamer; dioxygenase; gene regulation; hypoxanthine; noncoding RNA; urate

\section{INTRODUCTION}

Riboswitches that respond to metabolites or elemental ions are routinely used by many bacterial species to control the expression of genes coding for proteins that regulate metabolic or elemental ion homeostasis processes, or that make physiological changes in response to signaling molecules (Breaker 2011, 2012, 2018; Serganov and Nudler 2013; Sherwood and Henkin 2016; Lotz and Suess 2018). Over 45 different riboswitch classes have been experimentally validated, each using one or more characteristic aptamer domains to selectively bind a variety of fundamental metabolites or metal ions and control the expression of genes relevant to these ligands (McCown et al. 2017).

In addition, many classes of "orphan" riboswitch candidates (Barrick et al. 2004; Meyer et al. 2011; Weinberg et al. 2017a; Greenlee et al. 2018) have been discovered by using computational strategies to identify unusually well-conserved and structured RNAs located in the non-

Corresponding author: ronald.breaker@yale.edu

Article is online at http://www.rnajournal.org/cgi/doi/10.1261/rna. 075218.120. Freely available online through the RNA Open Access option. coding regions of some bacterial genomes. Both the number of orphan riboswitch candidates and the apparent diversity of these RNAs strongly suggest that far more riboswitch classes remain to be experimentally validated (Ames and Breaker 2010; McCown et al. 2017). If true, then the types of ligands sensed by riboswitch aptamers are likely to be far greater than is presently known. The discovery and validation of additional riboswitch classes and their associated regulatory networks will help reveal the functions of proteins whose activities were unknown or previously mischaracterized (e.g., see Baker et al. 2012; Nelson et al. 2017).

The NMT1 motif is an orphan riboswitch candidate that was previously identified through comparative sequence analysis as a structured, noncoding RNA of unknown function (Weinberg et al. 2017a). Representatives of this RNA motif are usually found immediately upstream of the open reading frames (ORFs) of variously annotated genes wherein the precise functions of their protein products remain uncharacterized. One class of genes commonly

(C) $2020 \mathrm{Yu}$ and Breaker This article, published in RNA, is available under a Creative Commons License (Attribution 4.0 International), as described at http://creativecommons.org/licenses/by/4.0/. 
associated with the NMT1 motif codes for proteins commonly annotated as NMT1/THI5-like. These annotations imply that the genes share similarity to the NMT1 gene of fungi, which codes for an enzyme involved in synthesis of the pyrimidine moiety of thiamin (McColl et al. 2003; Bale et al. 2010). There are already two validated riboswitch classes related to thiamin metabolism: one that senses the coenzyme form, thiamin pyrophosphate (TPP) (Mironov et al. 2002; Winkler et al. 2002) and another that senses the TPP precursor 4-amino-5-(hydroxymethyl)2-methylpyrimidine pyrophosphate (HMP-PP) (Atilho et al. 2019). These observations were used to formulate our initial hypothesis that NMT1 motif RNAs function as metabolite-binding riboswitches, perhaps for a ligand related to TPP metabolism.

However, further bioinformatic analysis of the NMT1/ THI5-like annotated genes associated with the RNA motif revealed that these genes were distinct from the NMT1 genes relevant to thiamin metabolism. Moreover, the various other genes associated with NMT1 motifs were similar to those encoding non-heme iron (II) dependent dioxygenases, alpha-ketoglutarate dependent dioxygenases, nucleoside transporters, and adenosine and guanine deaminases. These bioinformatic analyses thus implicated the genes associated with NMT1 motifs in purine degradation, rather than thiamin metabolism.

There are also various known riboswitch classes that sense purines and their biosynthetic intermediates, including guanine (Mandal et al. 2003), adenine (Mandal and Breaker 2004), ZTP (Kim et al. 2015), and phosphoribosyl pyrophosphate (PRPP) (Sherlock et al. 2018a). These riboswitch classes commonly regulate genes related to the production of purine nucleobases or purine transport. In addition, rare riboswitches for 2'-deoxyguanosine-5'-monophosphate (dGMP) regulate ribonucleotide reductase genes to control biosynthesis of purine DNA monomers (Kim et al. 2007; Weinberg et al. 2017b), and a riboswitch class also exists that senses 5 '-diphosphorylated adenosine nucleotides ADP and dADP to regulate NUDIX hydrolases (Sherlock et al. 2019). Finally, several riboswitch classes exist that sense signaling molecules derived from purine nucleotides, including c-di-GMP (Sudarsan et al. 2008; Lee et al. 2010), c-di-AMP (Nelson et al. 2013), c-AMP-GMP (Kellenberger et al. 2015; Nelson et al. 2015a), and ppGpp (Sherlock et al. 2018b). All these experimentally validated riboswitch classes provide abundant precedence for aptamers that sense purines and their metabolites, demonstrating that the bacterial domain of life extensively exploits RNA to monitor the concentrations of various purines and their natural derivatives. Previously, however, there were no known riboswitch classes that predominantly regulate enzymes involved in the degradation of purine nucleobases.

In the current study, we demonstrate that NMT1 motif RNAs selectively recognize oxidized purine nucleobases.
Structure activity relationship (SAR) data reveal that natural ligands xanthine and uric acid are bound most tightly. In addition, genetic analyses demonstrate that representatives of these RNAs, now renamed xanthine riboswitches, function as genetic "OFF" switches to repress the translation of mRNAs whose gene products would otherwise generate oxidized purines. These findings reveal that some bacterial cells monitor the levels of oxidized purines, presumably to avoid accumulating unnecessary or even toxic levels of these purine degradation waste products.

\section{RESULTS AND DISCUSSION}

\section{Genes associated with the NMT1 motif share similarity with dioxygenases involved in purine oxidation}

The consensus sequence and secondary structure model originally reported for NMT1 motif RNAs (Weinberg et al. 2017a) was updated after the identification of additional unique-sequence representatives. These additional RNAs were uncovered and subsequently examined by using comparative sequence analysis algorithms to search recently released bacterial genomic sequence data (see Materials and Methods for details). A total of 649 representatives have now been identified, which are widely distributed in alpha-, beta- and gamma-Proteobacteria.

The additional NMT1 motif representatives were examined to create an updated consensus model and to expand the available information regarding gene associations. The revised secondary structure model roughly conforms to a two-stem (P1 and P2) junction, wherein stem P2 forms a small internal bulge to divide the stem into two parts (P2a and P2b) (Fig. 1A). Key differences between the new consensus model and the previous model are supported by nucleotide covariation that is consistent with the formation of $\mathrm{P} 2 \mathrm{a}$, and by the absence of covariation evidence that led to the reduction in proposed length for the P1 stem. Most of the highly conserved nucleotides in the updated consensus model are located in the bulged nucleotides forming the junction between P1 and P2a ( $\mathrm{J} 1$ 2a), and likewise in the junctions J2b-2a and J2a-1. We speculated that these highly conserved nucleotides are likely to be critical for the formation of the ligand-binding aptamer structure.

Bioinformatic comparisons between representatives also yielded evidence for the common use of expression platforms that use sequestration of the ribosome binding site (RBS) of the adjacent open reading frame (ORF) (Supplemental Fig. S1). This type of translation control mechanism is frequently used by members of many riboswitch classes (Barrick and Breaker 2007; Breaker 2018). Almost no examples of intrinsic terminator stems were identified, which is consistent with previous observations (Weinberg et al. 2017a) and with the fact that NMT1 motif 


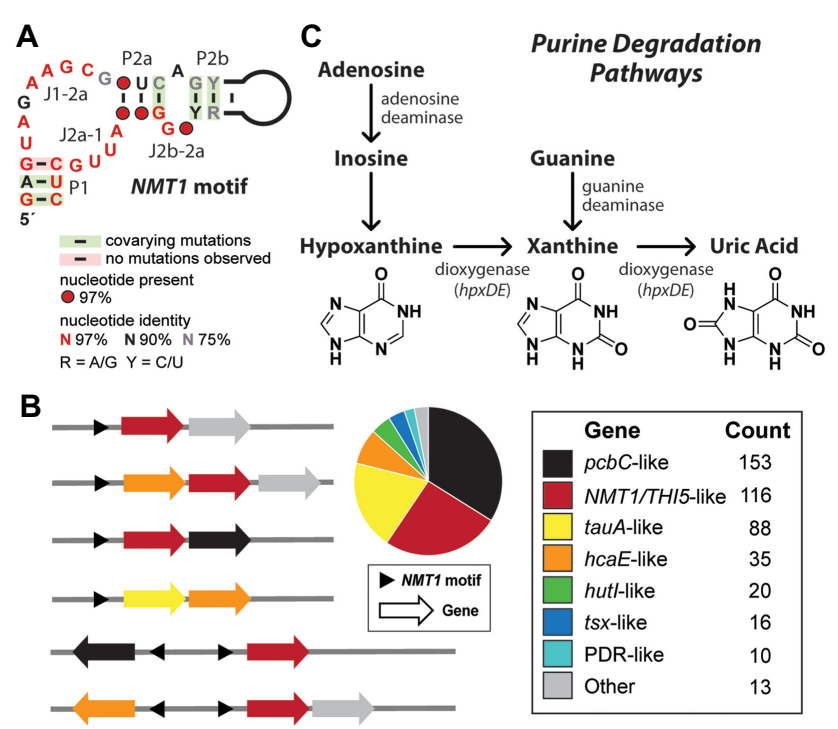

FIGURE 1. Characteristics of NMT1 motif RNAs. (A) Updated consensus sequence and secondary structure model for the NMT1 motif based on 649 unique representatives. (B) Downstream genetic contexts of NMT1 motif representatives. (Left) Representative genetic arrangements reflect diverse gene associations, including operons. (Right) The pie chart depicts the abundances of the gene located immediately downstream from NMT1 motif representatives. (C) Basic purine degradation and utilization pathways (Riva et al. 2008). Genes $h p x D$ and $h p x E$ code for known aromatic ring hydrolases.

RNAs are found exclusively in Proteobacteria. These species rarely utilize expression platforms with a direct transcription control mechanism (Barrick and Breaker 2007).

As described previously (Weinberg et al. 2017a), genes located immediately downstream from NMT1 motif representatives primarily belong to three gene types, which code for proteins with similarities either to isopenicillin $\mathrm{N}$ synthase and related dioxygenases ( $p c b C$ ), to NMT1/ THI5-like proteins (see above), or to ABC-type nitrate/sulfonate/bicarbonate transporters (tauA). Additional associated genes code for proteins similar to various dioxygenases (hcaE, PDR, DIOX_N), metallodependent amidohydrolases (hutl), amidohydrolases similar to 8-hydroxyguanine and guanine deaminases (PRK12393), nucleoside transporters (tsx), and in rare instances tRNA adenosine deaminases $(\operatorname{tad} A)$. Notably, these associated genes sometimes are found in series following an NMT1 motif RNA, indicating that some members of this candidate riboswitch class regulate an operon of genes related to the same metabolic process (Fig. 1B).

We further examined the genetic context of NMT1 motif RNAs by using NCBI Protein Blast on the associated genes that were annotated as dioxygenases. These dioxygenases were previously predicted to be Rieske non-heme iron aromatic-ring-hydroxylating enzymes or alpha-ketoglutarate dependent dioxygenases that share sequence similarity with the hpxD and hpxE genes cluster discovered in Klebsiella pneumoniae (Riva et al. 2008). In K. pneumo- niae, the hpxDE genes are involved in the oxidation of hypoxanthine to produce xanthine, and the oxidation of xanthine to yield uric acid (Fig. 1C; Riva et al. 2008). This additional information led us to pursue a hypothesis wherein the NMT1 motif architecture represents a highly conserved aptamer domain that selectively binds an oxidation product resulting from purine nucleotide degradation.

\section{NMT1 motif RNAs function as selective aptamers for xanthine and uric acid}

An RNA construct called 53 tauA (Fig. 2A) carrying 53 nt of the NMT1 motif representative associated with the tauA gene from Serratia plymuthica strain S13 was prepared by in vitro transcription. $5^{\prime}{ }^{32} \mathrm{P}$-labeled RNAs were then evaluated by using in-line probing, which is a method that provides information on the structural characteristics of RNA, including folding changes brought about by ligand binding (Soukup and Breaker 1999, Regulski and Breaker 2008). Initial examination of a diverse collection of purines revealed that modulation of the 53 tauA RNA structure is triggered by xanthine binding (Fig. 2B), which exhibits an apparent dissociation constant $\left(K_{D}\right)$ of $\sim 3.7$ $\mu \mathrm{M}$ (e.g., Fig. 2C) based on two repeat experiments. Similar results were obtained for uric acid $\left(K_{D} \sim 25 \mu \mathrm{M}\right)$, which differs from xanthine by oxidation of the $\mathrm{C} 8$ position of the purine ring (Supplemental Fig. S2).

Ligand binding by the aptamer is dependent on the presence of highly conserved nucleotides in the joining regions. This assessment was made by examining mutant versions of an extended aptamer construct carrying $68 \mathrm{nt}$ upstream of the tauA ORF from S. plymuthica S13 called 68 tauA (Fig. 3A). This extended construct was originally prepared and tested concurrently with the 53 tauA construct to reduce the experimental risk of testing only a single construct that might be trimmed too short or that might misfold. Mutations were arbitrarily tested using the longer of the two constructs.

Mutant construct M1 of the 68 tauA construct, which carries a single $A$ to $U$ mutation at position 12 that alters the nucleotide identity at an otherwise strictly conserved site, exhibits a complete loss of xanthine binding (Fig. 3B). Construct $\mathrm{M} 2$, which carries a $\mathrm{G}$ to $\mathrm{C}$ mutation at the invariant nucleotide position 38, likewise loses ligand binding function. Although there appears to be some modulation of the $\mathrm{M} 2$ construct in response to xanthine in the final lane (Fig. 3B), there are two reasons why we believe this does not reflect the normal function of the RNA. First, the banding pattern suggests that the structure adopted by the M2 construct is distinct from WT, and therefore the mutant construct is not expected to function normally. Second, the banding pattern changes are different than those for the unmodified construct. Given that this last lane has a very high concentration of xanthine, we 

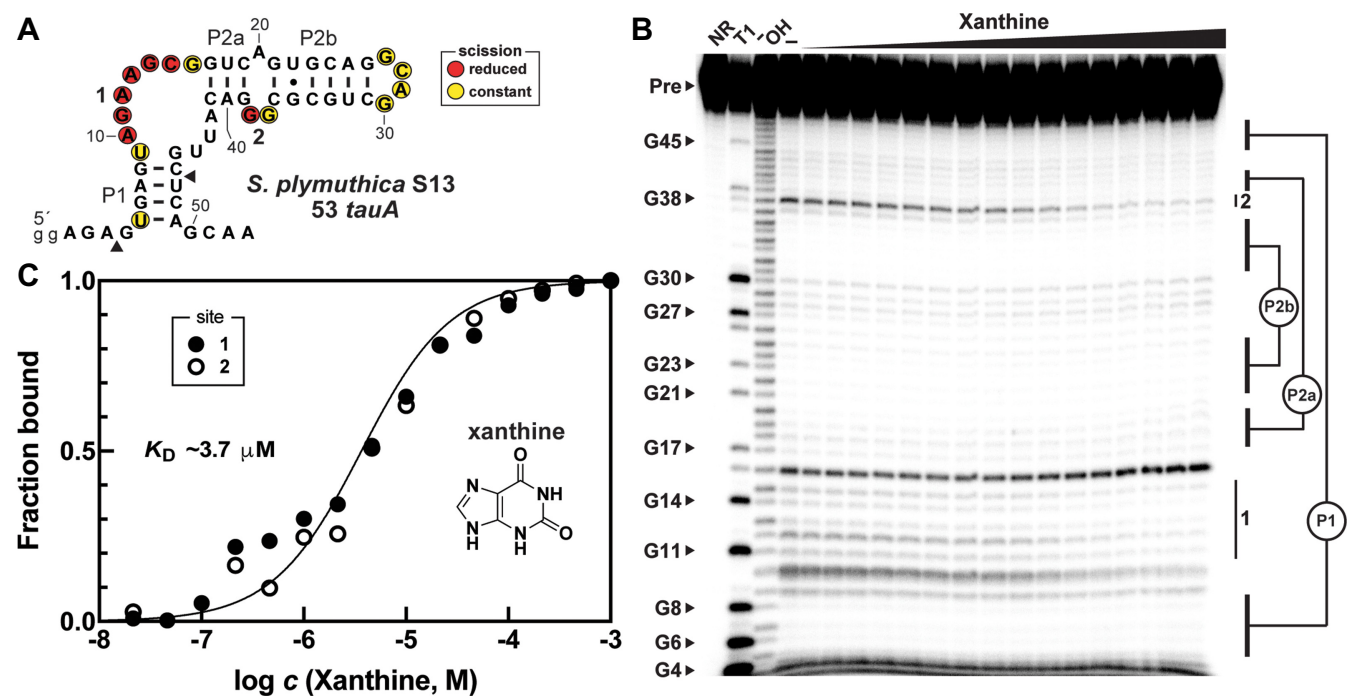

FIGURE 2. An NMT1 motif RNA binds xanthine. (A) Sequence and secondary structure model of the 53 tauA NMT1 motif RNA construct based on a representative from $S$. plymuthica $\mathrm{S} 13$, which is naturally located upstream of the tauA coding region. The $5^{\prime}$ terminus includes two guanosine nucleotides (lowercase letters) to improve in vitro transcription efficiency. Nucleotides whose phosphodiester linkages undergo scission during inline probing reactions (see the PAGE image in B) are encircled and undergo modulation as indicated. (B) PAGE autoradiogram of $5^{\prime 2}{ }^{32} \mathrm{P}-\mathrm{labeled} 53$ tauA RNAs subjected to in-line probing reactions without (-), or with a range $(10 \mathrm{nM}$ to $1 \mathrm{mM})$ of xanthine concentrations. Lanes $\mathrm{NR}$, $\mathrm{T} 1$, and ${ }^{-} \mathrm{OH}$ indicate RNAs subjected to no reaction, limited digestion with RNase T1 (cleaves after each G), and incomplete digestion under alkaline conditions (cleaves after every nucleotide), respectively. The RNA precursor (Pre) band and select bands generated via RNase T1 digestion are indicated. Regions 1 and 2 correspond to sites of the RNA that exhibit structural modulation upon addition of xanthine. (C) Plot of the fraction of RNA bound to ligand versus the logarithm of the concentration of xanthine, as determined based on quantification of band intensity changes at sites 1 and 2 of the single experiment depicted in $B$. A trendline with a $K_{\mathrm{D}}$ of $3.7 \mu \mathrm{M}\left(R^{2}=0.9515\right)$ generated from a four-parameter logistic fit with the following parameters (minimum value equal to 0 , maximum value equal to 1 and Hill coefficient equal to 1 ) is superimposed on the data points.

believe the changes are indicative of artifacts in banding patterns typically observed when high ligand concentrations nonspecifically affect in-line probing reactions.

Similar losses in ligand binding function were observed for constructs $M 1$ and $M 2$ when exposed to uric acid (Supplemental Fig. S3). Likewise, construct M3 (Fig. 3A), in which the stability of $\mathrm{P} 1$ is disrupted, also loses xanthine binding function (Supplemental Fig. S4). These results are consistent with our hypothesis that the highly conserved secondary structure and core of NMT1 motif RNAs forms a binding pocket for certain oxidized purines such as xanthine and uric acid.

To further examine the ligand binding characteristics of NMT1 motif RNAs, we conducted an SAR analysis by subjecting either the $53 \operatorname{tauA}$ (Fig. 2A) or the 68 tauA (Fig. 3A) RNA constructs to in-line probing analyses in the presence of various analogs of xanthine or other nucleobases and their derivatives. Each compound was initially tested at concentrations of $100 \mu \mathrm{M}$ and $1 \mathrm{mM}$ to screen for ligand binding (e.g. see Supplemental Fig. S5), and additional active compounds (Fig. 4A) were further analyzed to establish $K_{D}$ values (Fig. 4B; Supplemental Fig. S6). Most analogs tested, including the common natural pyrimidine nucleobases, are strongly rejected by the aptamers (Supplemental Figs. S5, S7), indicating that the ligand- binding pocket is highly selective for specific oxidized purine compounds.

The combined ligand binding data were then used to create a model depicting the proposed key molecular recognition contacts on xanthine (Fig. 4C). Notably, analogs of xanthine or uric acid that carry a single methyl group on the N1, N3, or N7 positions fail to associate with the aptamer (Supplemental Figs. S7, S8). Binding is also precluded by the presence of a methyl group on N9 of uric acid, or the presence of a ribose moiety at this same position on hypoxanthine (as the compound inosine). These results highlight the importance of these purine ring positions as molecular recognition contact points, although the additional bulk presented by methyl or ribose group appendages could cause a steric clash with the aptamer. Alteration of the carbonyl oxygen atoms on $\mathrm{C} 2$ and $\mathrm{C} 6$ is also disruptive, suggesting these oxygens might be involved in forming hydrogen bonds, or possibly forming contacts with $\mathrm{Mg}^{2+}$ ions as is common with structured RNAs such as riboswitches (Ferré-D'Amaré and Winkler 2011).

We also examined the ligand function of 8-azaxanthine, which is a small molecule inhibitor of uric acid oxidase, an enzyme that breaks down uric acid to 5-hydroxyisourate (Colloc'h et al. 1997; Ramazzina et al. 2006). This compound is bound with an affinity similar to xanthine and 


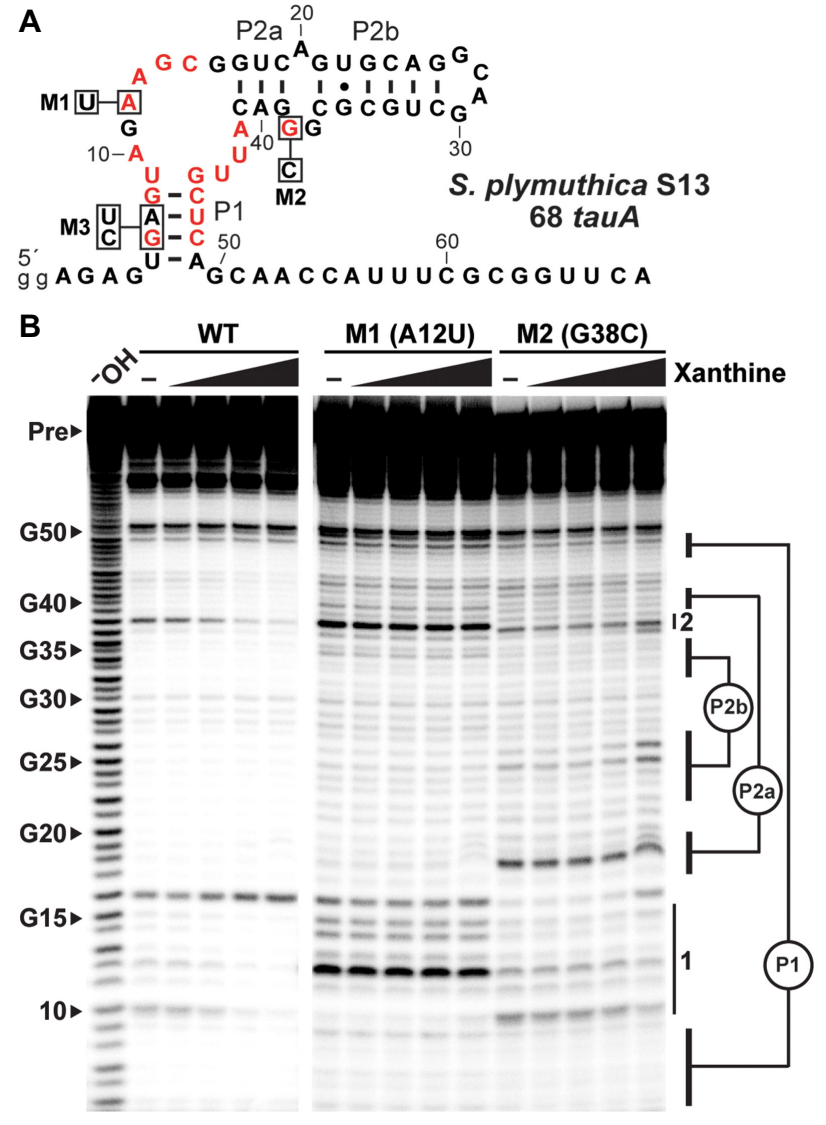

FIGURE 3. Mutations in the conserved core of an NMT1 motif RNA disrupts ligand binding. (A) Sequence and secondary model of the $68 \operatorname{tau} A$ construct with the location of mutations in constructs M1 and M2. Boxed nucleotides identify the mutation relative to the highly conserved WT nucleotides (red). (B) In-line probing data derived from subjecting $5^{\prime 32} \mathrm{P}$-labeled 68 tauA RNA to incubations in the absence $(-)$ or presence of xanthine as indicated. Arrowheads identify bands corresponding to the 5' RNA fragments produced by strand scission at the nucleotide positions as indicated. Other annotations are as described in the legend to Figure 2B.

uric acid, whereas some compounds with added bulk at position 8 of the purine ring exhibit some loss of binding affinity (Fig. 4A,B). These results suggest that the C8 position alone is of little importance for molecular recognition, although changes at this position might have some effect on the ability of the adjacent atoms to contact the aptamer. Also, there might be a modest steric effect that permits the aptamer to discriminate against ligands with certain chemical moieties attached to C8. Regardless, the current SAR data suggests that the aptamer exploits various contacts with xanthine and closely related analogs to form a highly selective binding pocket for natural purine oxidation products (Fig. 4C).

In the process of validating NMT1 motif RNAs as natural aptamers for xanthine and uric acid, we also tested other compounds chosen based on the genetic context, including those most relevant to the thiamin biosynthesis and histidine degradation pathways. However, we did not observe modulation of the 53 tauA RNA construct by these ligands at concentrations as high as $1 \mathrm{mM}$ (Supplemental Fig. S5). These findings again support our conclusion that NMT1 motif representatives function as high-affinity aptamers for xanthine and uric acid.

\section{Riboswitch-reporter fusion assays demonstrate that NMT1 motif RNAs function as genetic "OFF" switches}

To evaluate the possible genetic control functions of NMT1 motif RNAs, we created a construct for gene expression studies in Escherichia coli as a surrogate organism by fusing the NMT1 motif representative from Hydrogenophaga intermedia strain S1 to a $\beta$-galactosidase reporter gene (Fig. 5A). This NMT1 motif sequence is naturally associated with four genes that are commonly found with NMT1 motif representatives: two NMT1 genes, pcbC and tadA (Fig. 5A, inset). Specifically, the NMT1 and pcbC genes are relevant to purine oxidation as described above. The associated tadA gene is predicted to function as an adenosine deaminase, whose catalytic activity is expected to yield the oxidized nucleoside inosine. Therefore, the NMT1 motif example chosen for genetic analysis a typical representative of this candidate riboswitch class.

The $\mathrm{H}$. intermedia representative also was chosen in part because of the relatively short distance between the aptamer and the RBS. Specifically, a ribosome binding site (RBS) resides downstream from the conserved portion of the NMT1 motif, suggesting that the expression platform might involve the regulation of ribosome binding to the mRNA. Furthermore, there is no evidence for the presence of an intrinsic terminator stem (strong stem followed by a run of U nucleotides) (Wilson and von Hippel 1995; Yarnell and Roberts 1999) in this or other examples (Supplemental Fig. S1), which suggests that members of this riboswitch class do not directly use a transcription termination mechanism.

Two genetic reporter constructs were tested: a wild-type (WT) version and a mutant version (M4) that carries a single nucleotide change at the equivalent location to the 53 tauA M1 construct, which is known to cause a loss of ligand binding activity (Fig. 3A). In liquid media assays, levels of the WT riboswitch reporter gene expression were lowest under both rich media and minimal media growth conditions. In contrast, the M4 reporter construct exhibits higher gene expression under both rich and minimal media conditions, suggesting that the riboswitch ligand is present under both conditions, and that the RNA motif turns "OFF" translation of downstream genes (Fig. 5B).

All the genes regulated by the NMT1 motif are predicted to catalyze reactions on compounds that are earlier than xanthine and uric acid in the metabolic pathways for purine degradation. Therefore, the reporter assay results 
8-Azaxanthine Xanthine Uric Acid
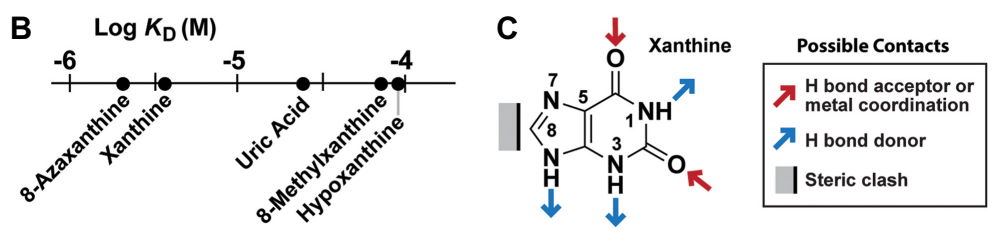

FIGURE 4. SAR data for ligand binding by an NMT1 motif RNA representative. (A) Chemical structures xanthine and various analogs that are bound by the 53 tauA RNA construct. (B) Plot of the $K_{D}$ values for various ligands. Compounds that failed to exhibit evidence of binding when tested at $1 \mathrm{mM}$ are depicted in Supplemental Figure S4, Supplemental Figure S6, and Supplemental Figure S7. (C) Proposed molecular recognition contacts based on the affinities observed for various ligand candidates tested.

are consistent with the genetic contexts of NMT1 motif RNAs. An excess of oxidized purine degradation products might need to trigger the repression of genes involved in the transport of nucleosides by nucleoside transporters, the deamination of purines by deaminases and amidohydrolases, and the oxidation of purines by dioxygenases, or the cell risks the toxic effects of these molecules. For example, uric acid is famously disruptive to biological systems due to its insolubility, and can cause joint and kidney damage in humans (Dalbeth et al. 2016).

\section{Concluding remarks}

Taken together, our findings support the hypothesis that NMT1 motif RNAs are selective riboswitches for oxidized purines such as xanthine and uric acid, and that they turn off genes whose expression would otherwise lead to the accumulation of potentially toxic levels of these compounds. However, it is notable that we do not observe genes related to oxidized purine degradation among the list of genes associated with this riboswitch class. We speculate that some species would have used representatives of this riboswitch class to activate genes involved in oxidized purine degradation or export if toxicity were the primary concern. This observation therefore suggests that cells might use this riboswitch class to mon-
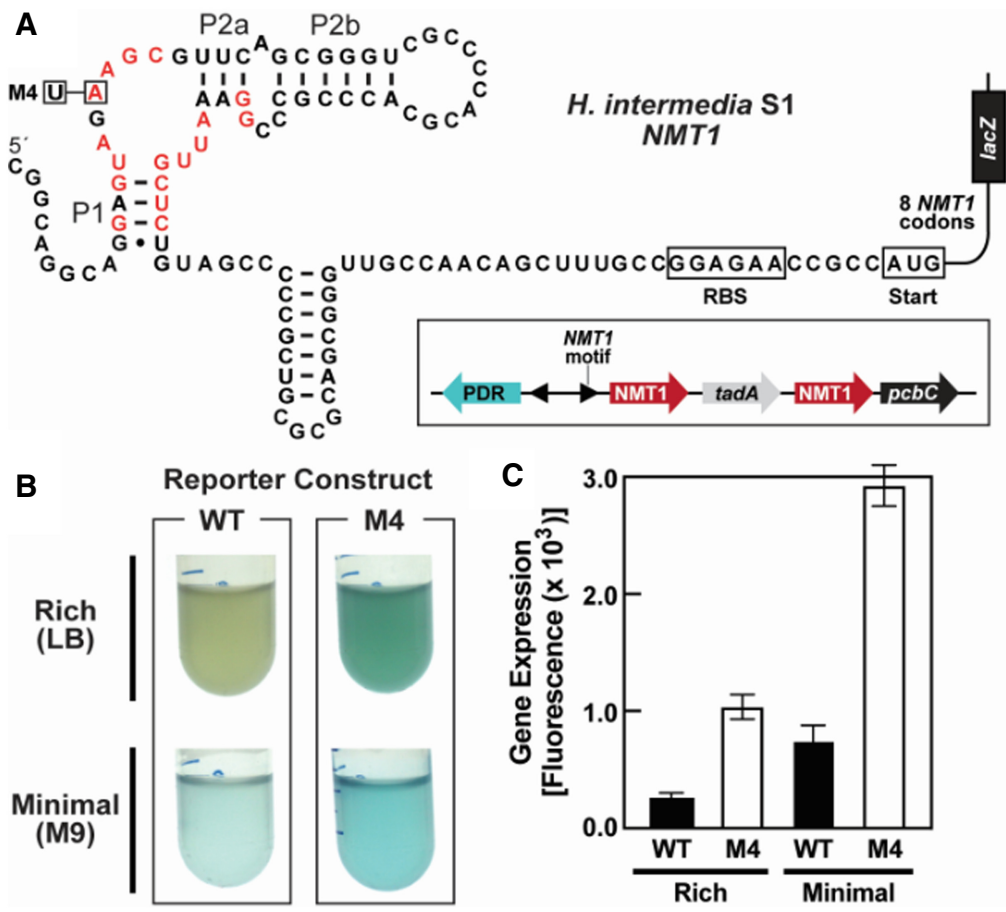

FIGURE 5. Reporter gene assays indicate that NMT1 motif RNAs are genetic "OFF" riboswitches. (A) Riboswitch-reporter fusion construct formed by linking the NMT1 motif RNA representative from $H$. intermedia S1 to lacZ gene. Red letters identify the highly conserved nucleotides from the NMT1 motif consensus model (Fig. 1A). (Inset) The bacterium H. intermedia S1 carries adjacent NMT1 motif representatives in opposing orientations. The right-most representative was used for the reporter construct. (B) Liquid media assays of E. coli cells harboring either the wild-type (WT) or the a single conserved-nucleotide mutant (M1) reporter construct. Cells were grown in rich (LB) or minimal (M9) media conditions in the presence of the $\beta$-galactosidase activity indicator x-gal. (C) Plot of reporter gene expression for the WT and M4 constructs of the $\mathrm{H}$. intermedia S1 riboswitch. Error bars represent the standard deviation for the measurements based on six replicate experiments. 
with this riboswitch class and other proteins with similar amino acid sequences provided clues that were sufficient to create a more promising collection of ligand candidates that could be tested.

Prior research had uncovered gene clusters in K. pneumoniae and $K$. oxytoca involved in hypoxanthine assimilation (Riva et al. 2008) and purine utilization (Pope et al. 2009), respectively. Although dioxygenases associated with NMT1 motif representatives share sequence similarity with alpha-ketogluatarate dependent dioxygenases, the precise functions of these proteins have not been established. The finding that NMT1 motif RNAs bind to xanthine and uric acid strongly supports the view that the dioxygenases will eventually be proven to operate on purine substrates. This hypothesis also is consistent with the fact that the dioxygenases associated with this riboswitch class are highly similar in sequence to dioxygenases encoded by hpxD and hpxE genes, which are known to be associated with purine oxidation pathways (Riva et al. 2008).

The strong binding affinities of NMT1 motif RNAs to 8azaxanthine, xanthine, and uric acid support the notion that the RNA binds to oxidized purines likely involved in the purine degradation pathway in these bacteria. However, we cannot be certain at this time whether members of this riboswitch have a specific preference in cells for the ligand that triggers gene control. It is possible that xanthine riboswitches respond to the accumulated pool of oxidized purines, or perhaps the natural ligand simply is the single oxidized purine form that accumulates the most. Regardless, our results will provide useful guidance as researchers perform further experiments to validate the functions of the proteins encoded by the riboswitch-controlled genes, which is warranted given the importance of purine metabolism in living systems.

\section{MATERIALS AND METHODS}

\section{Bioinformatics analyses}

Additional NMT1 motif RNAs (Supplemental File S1) were discovered by using the comparative sequence analysis algorithms CMfinder (Yao et al. 2006) and Infernal 1.1 (Nawrocki and Eddy 2013) as described previously (Weinberg et al. 2017a). The database examined was comprised of a complete set of genomic DNA sequences (RefSeq version 80) and microbial environmental sequence collections as described previously (Weinberg et al. 2017a). RNA sequence and secondary structure consensus models and covariation data were depicted by using R2R software (Weinberg and Breaker 2011), and manually examined to assess the data and adjust the depictions. Protein sequence homology was determined using NCBI Basic Local Alignment Search Tool (BLAST) (Altschul et al. 1990).

\section{Chemicals, biochemicals, and oligonucleotides}

Synthetic oligonucleotides (Supplemental Table S1) and most compounds used to conduct SAR analyses were obtained from
Sigma-Aldrich. Exceptions include 3- and 9-methyluric acid (Santa Cruz Biotechnology, Inc.), isoxanthopterine and 7-methyluric acid (Cayman Chemical Company), and 8-hydroxyguanine (Carbosynth). [ $\gamma^{32}$ P] ATP (specific activity: $6000 \mathrm{Ci} / \mathrm{mmol}$ ) was purchased from PerkinElmer. Genetic reporter constructs were designed (Supplemental Table S2) by the authors and subsequently purchased from GenScript VectorArt Gene Synthesis.

\section{RNA oligonucleotide preparation}

Synthetic DNA oligonucleotides templates with a T7 RNA polymerase (T7 RNAP) promoter on the $5^{\prime}$ terminus were transcribed, purified by $10 \%$ polyacrylamide gel electrophoresis (PAGE), and extracted, using protocols similar to those previously described (Chen et al. 2019).

To generate $5^{\prime}{ }^{32} \mathrm{P}$-labeled RNAs for in-line probing assays, 80 pmols of the RNA transcript was first dephosphorylated using rAPid alkaline phosphatase (Roche Life Sciences) following the manufacturer's protocol. Subsequently, 20 pmoles of the resulting RNA was ${ }^{32} \mathrm{P}$-radiolabeled at the $5^{\prime}$ terminus using T4 polynucleotide kinase in a $20 \mu \mathrm{L}$ reaction mixture consisting of $25 \mathrm{mM}$ CHES (pH 9.0 at $19^{\circ} \mathrm{C}$ ), $5 \mathrm{mM} \mathrm{MgCl}_{2}, 3 \mathrm{mM} \mathrm{DTT}$, and $20 \mu \mathrm{Ci}$ $\left[\gamma_{-}{ }^{32} \mathrm{P}\right]$ ATP. The reaction was incubated for $1 \mathrm{~h}$ at $37^{\circ} \mathrm{C}$. The resulting radiolabeled RNA was purified by denaturing 10\% PAGE and the desired RNA was extracted as noted above.

\section{In-line probing assays}

In-line probing assays were performed as previously described (Soukup and Breaker 1999; Regulski and Breaker 2008) with the following exceptions. Stock solutions of ligand candidates were prepared in $50 \mathrm{mM}$ aqueous sodium hydroxide. Reactions of 10 $\mu \mathrm{L}$ volume containing $\sim 125 \mathrm{nM}$ of $5^{\prime}{ }^{32} \mathrm{P}$-labeled RNA were incubated at $19^{\circ} \mathrm{C}$ for $36 \mathrm{~h}$ with the desired ligand in the presence of $100 \mathrm{mM}$ Tris- $\mathrm{HCl}\left(\mathrm{pH} 8.3\right.$ at $\left.23^{\circ} \mathrm{C}\right), 100 \mathrm{mM} \mathrm{KCl}$, and $20 \mathrm{mM}$ $\mathrm{MgCl}_{2}$. Denaturing 10\% PAGE was performed to resolve the reaction products. Binding curves and apparent $K_{D}$ values were estimated as previously described (Malkowski et al. 2019) using ImageQuant 5.1 (GE Healthcare Life Sciences) for quantitation and GraphPad Prism 8 for graphical analysis.

\section{Genetic reporter assays}

Genetic reporter constructs were designed with a thiC promoter from E. coli, followed by the NMT1 motif RNA representative from $\mathrm{H}$. intermedia strain $\mathrm{S} 1$, and included the first eight codons of the downstream ORF. This construct was inserted into 5' EcoRI and $3^{\prime}$ BamHI restriction sites of plasmid pRS414 (Simons et al. 1987) upstream and in-frame with a lacZ ( $\beta$ galactosidase) gene by GenScript VectorArt Gene Synthesis. WT and M4 reporter constructs in plasmid pRS414 were transformed into E. coli strain BW25113 from the Keio Collection (Baba et al. 2006) and grown overnight in Luria Broth (LB) at $37^{\circ} \mathrm{C}$ with modest shaking. The following day, cultures were diluted to an $\mathrm{OD}_{600}$ of 0.1 and grown in both rich (LB) and minimal (M9) media in the presence of $50 \mu \mathrm{g}$ $\mathrm{mL}^{-1}$ of X-gal (5-bromo-4-chloro-3-indolyl- $\beta$-D-galactopyranoside). Changes in culture color were recorded after $11 \mathrm{~h}$ incubations. 
Liquid $\beta$-galactosidase assays were performed to quantify the observations in media supplemented with X-gal. Reporter gene expression was quantified as described previously (Nelson et al. 2015b; Perkins et al. 2019) with the following differences. Cells were grown overnight in rich media (LB) or minimal media (M9) for $18 \mathrm{~h}$ at $37^{\circ} \mathrm{C}$ while shaking at $220 \mathrm{rpm} .80 \mu \mathrm{L}$ of a $1: 100$ diluted cell suspension $(990 \mu \mathrm{L}$ of fresh media with appropriate antibiotics plus $10 \mu \mathrm{L}$ of the previous culture) was applied in triplicate to individual wells on a Costar black 96-well clear-bottom plate and incubated for $18 \mathrm{~h}$. Following incubation, cell density and $\beta$-galactosidase activity measurements were performed as described elsewhere (Perkins et al. 2019).

\section{SUPPLEMENTAL MATERIAL}

Supplemental material is available for this article.

\section{ACKNOWLEDGMENTS}

We thank Felina Lenkeit (University of Konstanz) for collaborative efforts to assess initial ligand hypotheses. We also thank Narasimhan Sudarsan, Adam Roth, and other members of the Breaker Laboratory for helpful conversations, and Caroline Focht (Strobel Laboratory) for supplying purified HMP-PP. This work was supported by National Institutes of Health grant GM022778 to R.R.B. and by the Howard Hughes Medical Institute.

Received February 26, 2020; accepted April 1, 2020.

\section{REFERENCES}

Altschul SF, Gish W, Miller W, Myers EW, Lipman DJ. 1990. Basic local alignment search tool. J Mol Biol 215: 403-410. doi:10.1016/ S0022-2836(05)80360-2

Ames TD, Breaker RR. 2010. Bacterial riboswitch discovery and analysis. In The chemical biology of nucleic acids (ed. Mayer G), pp. 433-454. Wiley, Chichester, UK

Atilho RM, Arachchilage GM, Greenlee EB, Knecht KM, Breaker RR. 2019. A bacterial riboswitch class for the thiamin precursor HMPPP employs a terminator-embedded aptamer. Elife 8: e45210. doi:10.7554/eLife.45210

Baba T, Ara T, Hasegawa M, Takai Y, Okumura Y, Baba M, Datsenko KA, Tomita M, Wanner BL, Mori H. 2006. Construction of Escherichia coli K-12 in-frame, single-gene knockout mutants: the Keio collection. Mol Syst Biol 2: 2006.0008. doi:10.1038/ msb4100050

Baker JL, Sudarsan N, Weinberg Z, Roth A, Stockbridge RB, Breaker RR. 2012. Widespread genetic switches and toxicity resistance proteins for fluoride. Science 335: 233-235. doi:10.1126/sci ence. 1215063

Bale S, Rajashankar KR, Perry K, Begley TP, Ealick SE. 2010. HMP binding protein ThiY and HMP-P synthase THI5 are structural homologues. Biochemistry 49: 8929-8936. doi:10.1021/bi101209t

Barrick JE, Breaker RR. 2007. The distributions, mechanisms, and structures of metabolite-binding riboswitches. Genome Biol 8: R239. doi:10.1186/gb-2007-8-11-r239

Barrick JE, Corbino KA, Winkler WC, Nahvi A, Mandal M, Collins J, Lee M, Roth A, Sudarsan N, Jona I, et al. 2004. New RNA motifs suggest an expanded scope for riboswitches in bacterial genetic control. Proc Natl Acad Sci 101: 6421-6426. doi:10.1073/pnas .0308014101

Breaker RR. 2011. Prospects for riboswitch discovery and analysis. Mol Cell 43: 867-879. doi:10.1016/j.molcel.2011.08.024

Breaker RR. 2012. Riboswitches and the RNA world. Cold Spring Harb Perspect Biol 4: a003566. doi:10.1101/cshperspect.a003566

Breaker RR. 2018. Riboswitches and translation control. Cold Spring Harb Perspect Biol 10: a032797. doi:10.1101/cshperspect .a032797

Chen X, Mirihana Arachchilage G, Breaker RR. 2019. Biochemical validation of a second class of tetrahydrofolate riboswitches in bacteria. RNA 25: 1091-1097. doi:10.1261/rna.071829.119

Colloc'h N, Hajji ME, Bachet B, L'Hermite G, Schiltz M, Prangé T, Castro B, Mornon J-P. 1997. Crystal structure of the protein drug urate oxidase-inhibitor complex at 2.05 A resolution. Nat Struct Biol 4: 947-952. doi:10.1038/nsb1197-947

Dalbeth N, Merriman TR, Stamp LK. 2016. Gout. Lancet 388: 2039 2052. doi:10.1016/S0140-6736(16)00346-9

Ferré-D'Amaré AR, Winkler WC. 2011. The roles of metal ions in regulation by riboswitches. Met lons Life Sci 9: 141-173. doi:10.1039/ 9781849732512-00141

Greenlee EB, Stav S, Atilho RM, Brewer KI, Harris KA, Malkowski SN, Mirihana Arachchilage G, Perkins KR, Sherlock ME, Breaker RR. 2018. Challenges of ligand identification for the second wave of orphan riboswitch candidates. RNA Biol 15: 377-390. doi:10 .1080/15476286.2017.1403002

Kellenberger CA, Wilson SC, Hickey SF, Gonzalez TL, Su Y, Hallberg ZF, Brewer TF, lavarone AT, Carlson HK, Hsieh Y-F, et al. 2015. GEMM-I riboswitches from Geobacter sense the bacterial second messenger cyclic AMP-GMP. Proc Natl Acad Sci 112: 5383-5388. doi:10.1073/pnas.1419328112

Kim JN, Roth A, Breaker RR. 2007. Guanine riboswitch variants from Mesoplasma florum selectively recognize 2'-deoxyguanosine. Proc Natl Acad Sci 104: 16092-16097. doi:10.1073/pnas .0705884104

Kim PB, Nelson JW, Breaker RR. 2015. An ancient riboswitch class in bacteria regulates purine biosynthesis and one-carbon metabolism. Mol Cell 57: 317-328. doi:10.1016/j.molcel.2015.01.001

Lee ER, Baker JL, Weinberg Z, Sudarsan N, Breaker RR. 2010. An allosteric self-splicing ribozyme triggered by a bacterial second messenger. Science 329: 845-848. doi:10.1126/science.1190713

Lotz TS, Suess B. 2018. Small-molecule-binding riboswitches. Microbiol Spectr 6. doi:10.1128/microbiolspec. RWR-0025-2018

Malkowski SN, Spencer TCJ, Breaker RR. 2019. Evidence that the nadA motif is a bacterial riboswitch for the ubiquitous enzyme cofactor $\mathrm{NAD}^{+}$. RNA 25: 1616-1627. doi:10.1261/rna.072538.119

Mandal M, Breaker RR. 2004. Adenine riboswitch and gene activation by disruption of a transcription terminator. Nat Struct Mol Biol 11: 29-35. doi:10.1038/nsmb710

Mandal M, Boese B, Barrick JE, Winkler WC, Breaker RR. 2003. Riboswitches control fundamental biochemical pathways in Bacillus subtilis and other bacteria. Cell 113: 577-586. doi:10 .1016/S0092-8674(03)00391-X

McColl D, Valencia CA, Vierula PJ. 2003. Characterization and expression of the Neurospora crassa nmt-1 gene. Curr Genet 44: 216 223. doi:10.1007/s00294-003-0441-0

McCown PJ, Corbino KA, Stav S, Sherlock ME, Breaker RR. 2017. Riboswitch diversity and distribution. RNA 23: 995-1011. doi:10 $.1261 /$ rna.061234.117

Meyer MM, Hammond MC, Salinas Y, Roth A, Sudarsan N, Breaker RR. 2011. Challenges of ligand identification for riboswitch candidates. RNA Biol 8: 5-10. doi:10.4161/rna.8.1.13865

Mironov AS, Gusarov I, Rafikoc R, Lopez LE, Shatalin K, Kreneva RA, Perumov DA, Nudler E. 2002. Sensing small molecules by nascent 
RNA: a mechanism to control transcription in bacteria. Cell 111: 747-756. doi:10.1016/S0092-8674(02)01134-0

Nawrocki EP, Eddy SR. 2013. Infernal 1.1: 100-fold faster RNA homology searches. Bioinformatics 29: 2933-2935. doi:10.1093/bioinfor matics/btt509

Nelson JW, Sudarsan N, Furukawa K, Weinberg Z, Wang JW, Breaker RR. 2013. Riboswitches in eubacteria sense the second messenger c-di-AMP. Nat Chem Biol 9: 834-839. doi:10.1038/ nchembio. 1363

Nelson JW, Sudarsan N, Phillips GE, Stav S, Lünse CE, McCown PJ, Breaker RR. 2015a. Control of bacterial exoelectrogenesis by cAMP-GMP. Proc Natl Acad Sci 112: 5389-5394. doi:10.1073/ pnas. 1419264112

Nelson JW, Plummer MS, Blount KF, Ames TD, Breaker RR. 2015b. Small molecule fluoride toxicity agonists. Chem Biol 22: $527-$ 534. doi:10.1016/j.chembiol.2015.03.016

Nelson JW, Atilho RM, Sherlock ME, Stockbridge RB, Breaker RR. 2017. Metabolism of free guanidine in bacteria is regulated by a widespread riboswitch class. Mol Cell 65: 220-230. doi:10.1016/ j.molcel.2016.11.019

Perkins KR, Atilho RM, Moon MH, Breaker RR. 2019. Employing a ZTP riboswitch to detect bacterial folate biosynthesis inhibitors in a small molecule high-throughput screen. ACS Chem Biol 14: 2841-2850. doi:10.1021/acschembio.9b00713

Pope SC, Chen LL, Stewart V. 2009. Purine utilization by Klebsiella oxytoca M5a1: genes for ring-oxidizing and -opening enzymes. $J$ Bacteriol 191: 1006-1017. doi:10.1128/JB.01281-08

Ramazzina I, Folli C, Secchi A, Berni R, Percudani R. 2006. Completing the uric acid degradation pathway through phylogenetic comparison of whole genomes. Nat Chem Biol 2: 144-148. doi:10.1038/ nchembio768

Regulski EE, Breaker RR. 2008. In-line probing analysis of riboswitches. Methods Mol Biol 419: 53-67. doi:10.1007/978-159745-033-1_4

Riva L, Badia J, Aguilar J, Bender R, Baldoma L. 2008. The hpx genetic system for hypoxanthine assimilation as a nitrogen source in Klebsiella pneumoniae: gene organization and transcriptional regulation. J Bacteriol 190: 7892-7903. doi:10.1128/JB.01022-08

Serganov A, Nudler E. 2013. A decade of riboswitches. Cell 152: 1724. doi:10.1016/j.cell.2012.12.024

Sherlock MES, Sudarsan N, Breaker RR. 2018a. Riboswitches for the alarmone ppGpp expand the collection of RNA-based dignaling systems. Proc Natl Acad Sci 115: 6052-6057. doi:10.1073/pnas .1720406115
Sherlock MES, Sudarsan N, Stav S, Breaker RR. 2018b. Tandem riboswitches form a natural Boolean logic gate to control purine metabolism in bacteria. Elife 7: e33908. doi:10.7554/eLife.33908

Sherlock MES, Sadeeshkumar H, Breaker RR. 2019. Variant bacterial riboswitches associated with nucleotide hydrolase genes sense nucleoside diphosphates. Biochemistry 58: 401-410. doi:10 .1021/acs.biochem.8b00617

Sherwood AV, Henkin TM. 2016. Riboswitch-mediated gene regulation: novel RNA architectures dictate gene expression responses. Annu Rev Microbiol 70: 361-374. doi:10.1146/annurev-micro091014-104306

Simons RW, Houman F, Kleckner N. 1987. Improved single and multicopy lac-based cloning vectors for protein and operon fusions. Gene 53: 85-96. doi:10.1016/0378-1119(87)90095-3

Soukup GA, Breaker RR. 1999. Relationship between internucleotide linkage geometry and the stability of RNA. RNA 5: 1308-1325. doi:10.1017/S1355838299990891

Sudarsan N, Lee ER, Weinberg Z, Moy RH, Kim JN, Link KH, Breaker RR. 2008. Riboswitches in eubacteria sense the second messenger cyclic di-GMP. Science 321: 411-413. doi:10.1126/sci ence.1159519

Weinberg Z, Breaker RR. 2011. R2R - software to speed the depiction of aesthetic consensus RNA secondary structures. BMC Bioinformatics 12: 3. doi:10.1186/1471-2105-12-3

Weinberg Z, Lunse CE, Corbino KA, Ames TD, Nelson JW, Roth A, Perkins KR, Sherlock ME, Breaker RR. 2017a. Detection of 224 candidate structured RNAs by comparative analysis of specific subsets of intergenic regions. Nucleic Acids Res 18: 10811-10823. doi:10 .1093/nar/gkx699

Weinberg Z, Nelson JW, Lünse CE, Sherlock ME, Breaker RR. 2017b. Bioinformatic analysis of riboswitch structures uncovers variant classes with altered ligand specificity. Proc Natl Acad Sci 114: E2077-E2085. doi:10.1073/pnas.1619581114

Wilson KS, von Hippel PH. 1995. Transcription termination at intrinsic terminators: the role of the RNA hairpin. Proc Natl Acad Sci 92: 8793-8797. doi:10.1073/pnas.92.19.8793

Winkler W, Nahvi A, Breaker RR. 2002. Thiamine derivatives bind messenger RNAs directly to regulate bacterial gene expression. Nature 419: 952-956. doi:10.1038/nature01145

Yao Z, Weinberg Z, Ruzzo WL. 2006. CMfinder - a covariance model based RNA motif finding algorithm. Bioinformatics 22: 445-452. doi:10.1093/bioinformatics/btk008

Yarnell WS, Roberts JW. 1999. Mechanism of intrinsic transcription termination and antitermination. Science 284: 611-615. doi:10 $.1126 /$ science. 284.5414 .611 

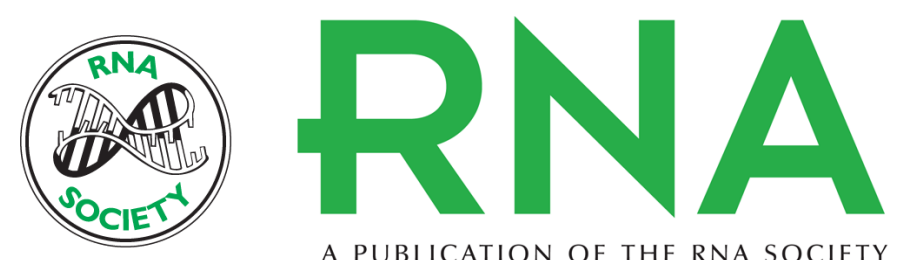

A PUBLICATION OF THE RNA SOCIETY

\section{A bacterial riboswitch class senses xanthine and uric acid to regulate genes associated with purine oxidation}

Diane Yu and Ronald R. Breaker

RNA 2020 26: 960-968 originally published online April 28, 2020

Access the most recent version at doi:10.1261/rna.075218.120

\section{Supplemental http://rnajournal.cshlp.org/content/suppl/2020/04/28/rna.075218.120.DC1 Material}

References This article cites 52 articles, 19 of which can be accessed free at: http://rnajournal.cshlp.org/content/26/8/960.full.html\#ref-list-1

Open Access Freely available online through the RNA Open Access option.

Creative This article, published in $R N A$, is available under a Creative Commons License Commons (Attribution 4.0 International), as described at

License http://creativecommons.org/licenses/by/4.0/.

Email Alerting Receive free email alerts when new articles cite this article - sign up in the box at the Service top right corner of the article or click here.

\section{|||||||| Providing Precise Solutions for your research.}

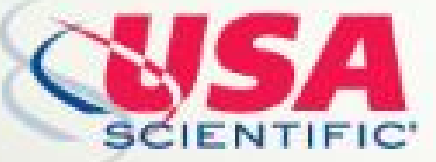

To subscribe to RNA go to:

http://rnajournal.cshlp.org/subscriptions

(C) 2020 Yu and Breaker; Published by Cold Spring Harbor Laboratory Press for the RNA Society 